\title{
EDITORIAL \\ Endonasal neurosurgery during the COVID-19 pandemic: the Singapore perspective
}

\author{
Yu Tung Lo, MBBS, MRCS, ${ }^{1}$ Neville Wei Yang Teo, MBBS, MRCS, MMed, ${ }^{2}$ and \\ Beng Ti Ang, MBBS, FRCSEd(SN) ${ }^{1}$
}

Departments of ${ }^{1}$ Neurosurgery and ${ }^{2}$ Otolaryngology, Singapore General Hospital, Singapore

$\mathrm{S}$ INGAPORE was among the first nations to be affected by the COVID-19 pandemic, with our first case reported on January $23,2020 .{ }^{1}$ Up until mid-February, Singapore reported the highest number of COVID-19 cases outside of China. ${ }^{2}$ Singapore has adopted a risk calibration and mitigation approach, which broadly speaking consists of the following: scaled screening in designated community clinics; targeted testing of suspected cases; extensive contact tracing of confirmed cases; a combination of isolation ("Stay-Home Notices") and quarantine measures (in supervised, designated venues) for high-risk individuals such as returnees from high-prevalence countries and individuals identified through contact tracing; social distancing measures; and free diagnostic tests and treatment in public hospitals for local residents. At present, the prevalence of community transmission in Singapore remains low, and the risk of transmission in the local asymptomatic population without relevant travel history is correspondingly low.

In neurosurgery, aerosol-generating procedures (AGPs) such as endonasal and anterior skull base surgery present the highest risk for COVID-19 exposure. Endonasal AGPs theoretically present a higher risk of viral exposure than intubation due to the aggressive disruption of potentially virus-containing mucosa. The duration of exposure for these procedures is also longer than during intubation. Common high-risk AGPs in neurosurgery include endoscopic endonasal pituitary surgery as well as anterior skull base procedures with the potential to breach the paranasal sinuses. Viral shedding has been found to be higher in the upper respiratory tract and, once aerosolized, virus particles remain airborne for more than 3 hours..$^{3,4} \mathrm{~A}$ high rate of infection has been reported among ear, nose, and throat (ENT) surgeons performing upper airway procedures. ${ }^{5}$ Endonasal surgery with the use of power tools should mandate the use of adequate protection due to potentially prolonged exposure to a high viral load. Neurosurgery and ENT departments across the nation have jointly developed advisories for these AGPs, in consultation with the Infec- tious Disease and Anesthesia Departments, and the hospitals' Surgical Division Committee.

\section{Preoperative COVID-19 Screening}

Prior to surgery and admission, for both outpatients and those transferring from another hospital, a comprehensive screening questionnaire is administered to review a list of symptoms and exposure history to identify patients who may be infected. Routine preoperative chest radiography is performed in all patients, and any suspicious findings are discussed with a dedicated on-call COVID-19 Infectious Disease team.

All patients who require elective high-risk surgery (defined as surgery that transgresses the upper airways) should undergo a routine preoperative swab for $\mathrm{CO}$ VID-19 the day before surgery, regardless of risk status. Once a patient tests negative for COVID-19, an N95 mask, eye protection (goggles and/or full face shield), and standard personal protective equipment (PPE; i.e., gown and gloves) will suffice for AGPs. Surgery for those who test positive for COVID-19 should be postponed until test results are negative, where possible. However, due to laboratory constraints in the face of the extremely large number of suspect cases, the capacity for routine swabs is not always available.

\section{COVID-19-Positive Patients}

Informal UK and US advisories have recommended the use of powered, air-purifying respirators (PAPRs) for all operating room staff during conduct of high-risk AGPs, and have suggested that an N95 mask and eye protection may not offer full protection. This was based on reports from Wuhan, China, that there was a high infection rate among ENT surgeons, and that the use of PAPRs anecdotally reduced the rate of transmission. A caveat was that the reports were not specific about whether the transmissions occurred during the actual surgery or during other perioperative phases (e.g., intubation, extubation, postop- 


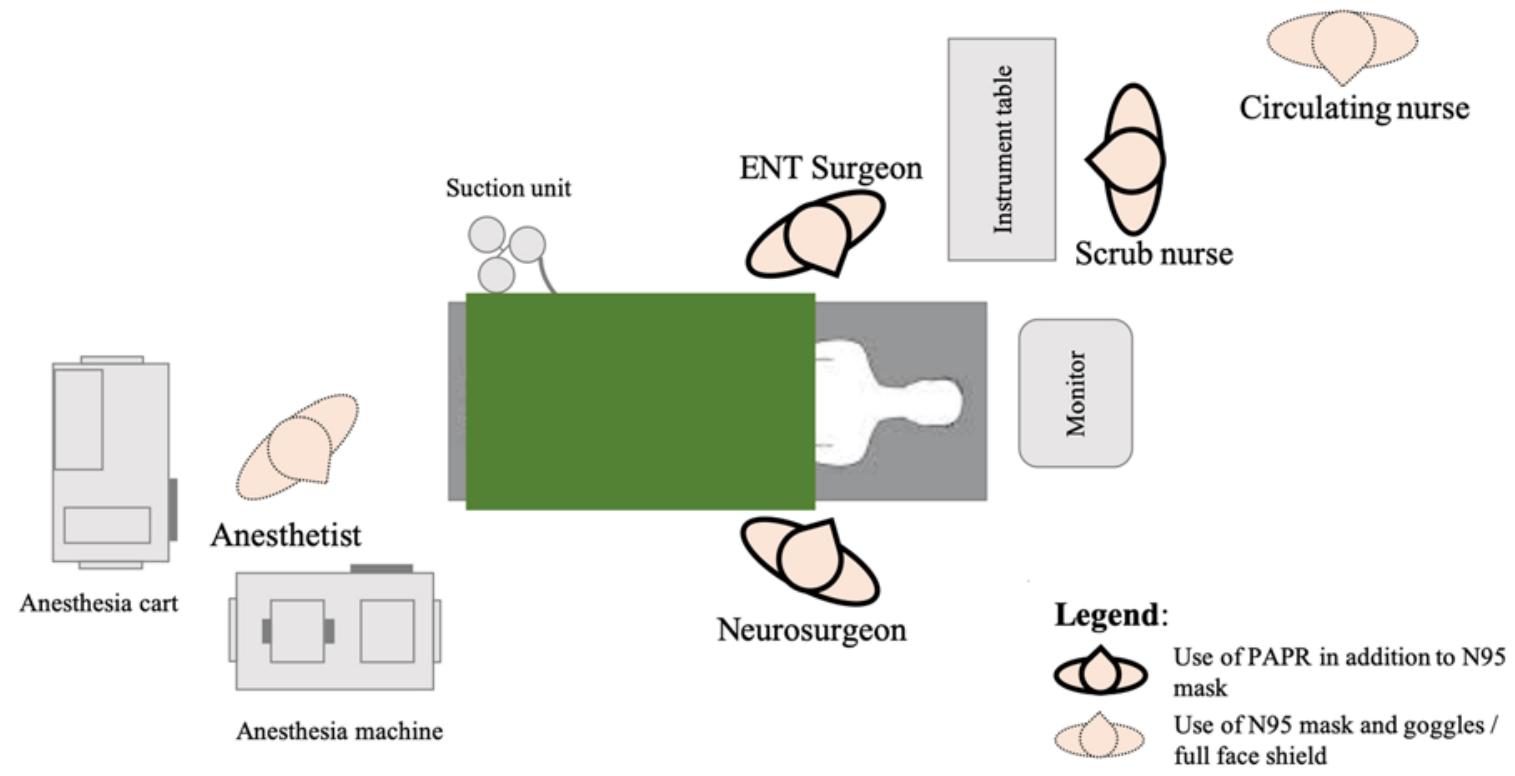

FIG. 1. Schema of operating room disposition during conduct of endonasal surgery for suspected COVID-19 cases. The operating surgeons and scrub nurse should don PAPRs, because they work in the immediate vicinity of the patient, whereas other personnel should don N95 masks, goggles, and standard PPE (gown and gloves). The anesthetist dons a PAPR during intubation and extubation but wears an N95 mask, goggles, and standard PPE during the surgery. Figure is available in color online only.

erative period). Our local consensus from Infectious Disease input is that for AGPs, even in COVID-19-positive patients, a properly fitted, molded, and handled N95 mask; eye protection (goggles/full face shield); and standard PPE (gown and gloves) should be adequate.${ }^{6,7}$ Nevertheless, because there is uncertainty over the exact mechanisms of virus transmission, degree of aerosolization, amount of viral load exposure, and lack of data on appropriate PPE in endonasal AGPs, a more cautious stance was adopted in which the entire surgical and anesthetic team would don PAPRs during the surgery, with the exception of the circulating nurse and operating theater attendant. Intraoperatively, the use of power instruments is avoided as much as is feasible without compromising the surgical exposure - using rongeurs and chisels instead. We also avoid the use of nasal packing or pledgets, which will need to be removed in the postoperative phase, because removal may induce coughing or gagging.

\section{Patients Whose COVID-19 Status Is Unknown}

Personal protective measures for patients with unknown COVID-19 status generated more debate. The safety of operating surgeons should be placed at the highest priority, although current resource limitation (or potential for such in the future) should also be taken into consideration to achieve a balance. Where possible, elective endonasal surgeries that are aerosol-generating should be deferred. For patients who do require urgent surgery, they should be assumed to be COVID-19-positive until proven otherwise, and managed accordingly. In accordance with this, the minimum PPE worn should be an N95 mask with goggles and standard PPE, but we recommend the use of PAPRs (where feasible and available) for the surgical team due to their closest proximity to the patient (Figs. 1 and
2). This is to mitigate the low but possible risk of operating on asymptomatic COVID-19-positive patients, which will increase as community prevalence increases, and in the absence of preoperative COVID-19 testing. For procedures in which headlight and loupes are required, alternative models of the PAPR such as the CleanSpace HALO may be used.

Aerosolization from the use of power instruments in contact with blood or bone, not just respiratory mucosa, has been postulated to present a small risk of viral transmission. One percent of blood specimens in a Chinese series contained detectable viral RNA. ${ }^{8}$ These considerations have wider implications for the rest of neurosurgery

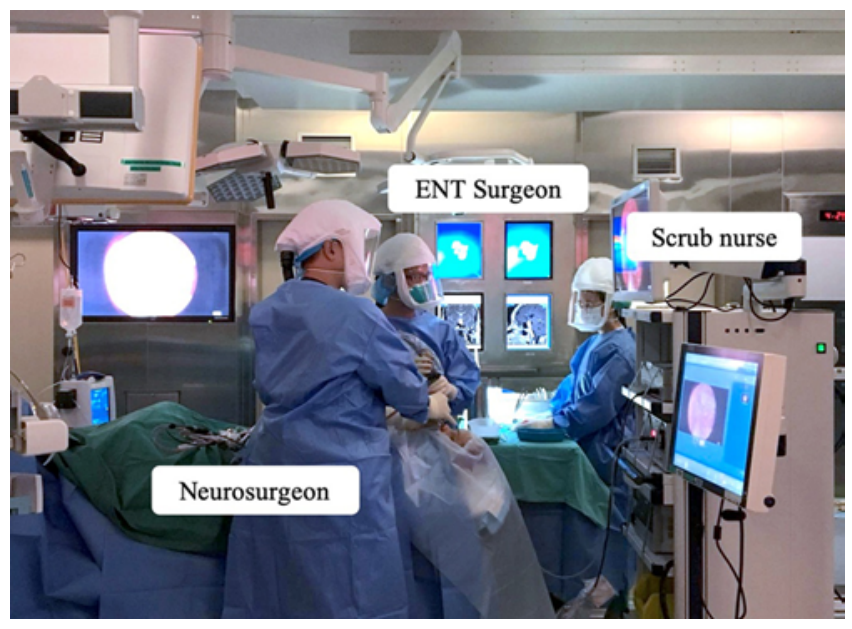

FIG. 2. Photograph showing implementation of PPE in the operating room. Figure is available in color online only. 
and other surgical specialties. Given finite resources, extensive testing and/or use of PPE will not be practical. All elective procedures should be postponed to minimize this theoretical risk as much as possible. Power drills should be irrigated more meticulously to minimize aerosolization.

\section{Future Directions}

The COVID-19 pandemic is an evolving situation and guidelines should change in light of new evidence in the future. Preparations should be made for the future should COVID-19 turn endemic. The total number of expected AGPs needs to be forecast within an institution to determine the required number of PAPR units. The measures proposed should be proportional to the level of risk, and take into consideration country-specific resource constraints. Most importantly, in the long haul, there is a need for healthcare workers to feel safe psychologically and to work in a safe environment.

https://thejns.org/doi/abs/10.3171/2020.4.JNS201036

\section{References}

1. Pung R, Chiew CJ, Young BE, et al. Investigation of three clusters of COVID-19 in Singapore: implications for surveillance and response measures. Lancet. 2020;395(10229):1039-1046.

2. World Health Organization. Coronavirus Disease (COVID-2019) Situation Reports. Accessed April 2, 2020. https://www.who.int/emergencies/diseases/novelcoronavirus-2019/situation-reports

3. Zou L, Ruan F, Huang M, et al. SARS-CoV-2 viral load in upper respiratory specimens of infected patients. $N$ Engl $J$ Med. 2020;382(12):1177-1179.
4. van Doremalen N, Bushmaker T, Morris DH, et al. Aerosol and surface stability of SARS-CoV-2 as compared with SARS-CoV-1. N Engl J Med. Published online March 17, 2020. doi:10.1056/nejmc2004973

5. Europe's doctors repeat errors made in Wuhan, China medics say. Bloomberg News. March 17, 2020. Accessed April 2, 2020. https://www.bloomberg.com/news/articles/2020-03-17/ europe-s-doctors-getting-sick-like-in-wuhan-chinesedoctors-say

6. World Health Organization. Rational use of personal protective equipment (PPE) for coronavirus disease (COVID-19): interim guidance. March 19, 2020. Accessed April 2, 2020. https://apps.who.int/iris/handle/10665/331498

7. Ng K, Poon BH, Kiat Puar TH, et al. COVID-19 and the risk to health care workers: a case report. Ann Intern Med. Published online March 16, 2020. doi:10.7326/L20-0175

8. Wang W, Xu Y, Gao R, et al. Detection of SARS-CoV-2 in different types of clinical specimens. JAMA. Published online March 11, 2020. doi:10.1001/jama.2020.3786

\section{Disclosures}

The authors report no conflict of interest.

\section{Correspondence}

Beng Ti Ang: ang.beng.ti@singhealth.com.sg.

\section{INCLUDE WHEN CITING}

Published online April 17, 2020; DOI: 10.3171/2020.4.JNS201036. 